\title{
Catalpol downregulates vascular endothelial-cadherin expression and induces vascular hyperpermeability
}

\author{
CAIQING ZHANG ${ }^{1}$, QINGFA LIU ${ }^{1}$, FENGYUN DONG ${ }^{2}$, LIQUN $^{2}{ }^{2}$, JUAN DU $^{2}$, QI XIE ${ }^{2}$, \\ HESHENG HU ${ }^{3}$, SUHUA YAN ${ }^{3}$, XIA ZHOU ${ }^{4}$, CHANGSHENG LI $^{4}$, CORRINNE G. LOBE ${ }^{5}$ and JU LIU ${ }^{2}$ \\ ${ }^{1}$ Department of Respiratory Diseases; ${ }^{2}$ Medical Research Center; ${ }^{3}$ Department of Cardiology; \\ ${ }^{4}$ Department of Traditional Chinese Medicine, Shandong Provincial Qianfoshan Hospital, Shandong University, \\ Jinan, Shandong 250014, P.R. China; ${ }^{5}$ Miami Mice Research Corp., Toronto, ON M5G 1L7, Canada
}

Received December 22, 2014; Accepted October 22, 2015

DOI: $10.3892 / \mathrm{mmr} .2015 .4522$

\begin{abstract}
Catalpol, an iridiod glucoside isolated from Rehmannia glutinosa, has been reported to possess anti-inflammatory properties. However, the molecular mechanisms underlying this effect have not been fully elucidated. This study aimed to investigate the effects of catalpol on vascular permeability. Using Transwell permeability assays and measurements of trans-endothelial electrical resistance (TEER), it was demonstrated that $1 \mathrm{mM}$ catalpol induces a significant increase in the permeability of the monolayers of human umbilical vein endothelial cells (HUVECs). Western blotting and immunofluorescence demonstrated that catalpol inhibits the expression of vascular endothelial (VE)-cadherin, the key component of adherens junctions, but not occludin, the major constituent of tight junctions. In addition, catalpol inhibits the ETS transcription factor ERG, a positive regulator of VE-cadherin. Knockdown of ERG expression compromised the catalpol-induced reduction of TEER in HUVECs. The present study revealed a novel effect of catalpol on vascular permeability and gave insight into the multifaceted roles of catalpol in inflammation.
\end{abstract}

\section{Introduction}

Vascular inflammation is crucial in a diverse group of diseases, including sepsis, atherosclerosis, diabetes and rheumatoid arthritis $(1,2)$. Once an inflammatory response is activated, circulating leukocytes (primarily monocytes and T lymphocytes) migrate across the vascular wall (1). The vascular endothelium responds with increased endothelial cell permeability, which enables the passage of plasma proteins and leukocytes from the capillary lumen to the subendothelial tissues,

Correspondence to: Professor Ju Liu, Medical Research Center, Shandong Provincial Qianfoshan Hospital, Shandong University, 16766 Jingshi Road, Jinan, Shandong 250014, P.R. China

E-mail: ju.liu@sdu.edu.cn

Key words: catalpol, endothelial cells, permeability, vascular endothelial-cadherin, ETS-related gene triggering further tissue damage (3). Vascular permeability is essential for the homeostasis of normal tissues, and hyperpermeability of vascular tissue is an important characteristic of inflammation.

The permeability properties of the endothelium are dynamically regulated by a vascular barrier, which is primarily formed by adherens junctions and tight junctions between endothelial cells (4). Adherens junctions are composed of membrane spanning vascular endothelial (VE)-cadherins, which interact with VE-cadherins expressed on neighboring cells via a homotropic mechanism in order to restrict paracellular permeability (5). The cytoplasmic tail of VE-cadherin binds to $\beta$-catenin linked to the cytoskeleton. Tight junctions are comprised of a branching network of sealing strands consisting of integral membrane-spanning proteins, such as occludin, which is directly linked to the actin cytoskeleton and mediates a series of cellular processes (6). During inflammation, proinflammantory cytokines, such as tumor necrosis factor (TNF)- $\alpha$ and interferon (IFN)- $\gamma$, induce the downregulation or re-distribution of the junctional proteins, leading to the breakdown of the vascular barrier (4).

Catalpol is an iridoid glucoside isolated from the root of Rehmannia glutinosa (7). A recent study has demonstrated the cardioprotective and anti-inflammatory properties of catalpol (8). In mouse models, catalpol protects against lipopolysaccharide-induced acute lung injury via the suppression of TNF- $\alpha$, interleukin (IL)-1 $\beta$, IL-4 and IL-6 (9). Catalpol also protects against cerebral ischemia/reperfusion injury by reducing free radicals and lipid peroxidation (10). In addition, catalpol suppresses inflammation-induced toxicity in dopaminergic neurons (11). However, the underlying mechanisms of its effects on inflammation have not been fully elucidated.

The present study examined the effects of catalpol on vascular permeability. Using real-time intercellular resistance analysis, it was demonstrated that catalpol induces an increase in the permeability of monolayers of human umbilical vein endothelial cells (HUVECs). In addition, the effects of catalpol on the expression of VE-cadherin, which is regulated by the ETS transcriptional factor ERG, was examined. This study identified a novel effect of catalpol on vascular permeability and provides a basis for catalpol-related drug development. 
Table I. Primer sequences.

\begin{tabular}{|c|c|c|c|}
\hline Gene & Sequence & Size (bp) & $\operatorname{Tm}\left({ }^{\circ} \mathrm{C}\right)$ \\
\hline \multicolumn{4}{|l|}{$\mathrm{CDH} 5$} \\
\hline Sense & GCGACTACCAGGACGCTTTCA & 150 & 60.5 \\
\hline Antisense & CATGTATCGGAGGTCGATGGTG & & \\
\hline \multicolumn{4}{|l|}{ OCLN } \\
\hline Sense & GAAGCCAAACCTCTGTGAGC & 227 & 59.0 \\
\hline Antisense & GAAGACATCGTCTGGGGTGT & & \\
\hline \multicolumn{4}{|l|}{$E R G$} \\
\hline Sense & TCTTGGACCAACAAGTAGCC & 151 & 57.5 \\
\hline Antisense & GTCGGGATCCGTCATCTTG & & \\
\hline \multicolumn{4}{|l|}{ GAPDH } \\
\hline Sense & TGATGACATCAAGAAGGTGGTGAAG & 240 & 57.9 \\
\hline Antisense & TCCTTGGAGGCCATGTGGGCCAT & & \\
\hline
\end{tabular}

\section{Materials and methods}

Cell culture. HUVECs (American Type Culture Collection, Manassas, VA, USA) were cultured in EBM2 basal endothelial cell medium supplemented with the EGM-2-MV bullet kit (Lonza, Basel, Switzerland) and antibiotics (100 IU/ml penicillin and $100 \mu \mathrm{g} / \mathrm{ml}$ streptomycin; Thermo Fisher Scientific, Inc., Waltham, MA, USA). The cells were cultured in humidified air at $37^{\circ} \mathrm{C}$ with $5 \% \mathrm{CO}_{2}$. Catalpol $(1 \mathrm{M})$ was purchased from Sigma-Aldrich (St. Louis, MO, USA) and dissolved in dimethyl sulfoxide (Beijing Solarbio Science \& Technology Co., Ltd., Beijing, China).

Fluorescein isothiocyanate (FITC)-Dextran Transwell assay. HUVEC monolayers were plated on the Transwell insert (Corning Incorporated, New York, NY, USA) and cultured until $100 \%$ confluent. Following pre-treatment with catalpol (0. 0.01, 0.1, $1 \mathrm{mM}$ ) at different concentrations, FITC-Dextran (Invitrogen; Thermo Fisher Scientific, Inc.) was added to the top chamber. Samples were removed from the bottom chamber after $24 \mathrm{~h}$ and read in a fluorometer (SpectraMax M3; Molecular Devices, Sunnyvale, CA, USA) at an excitation of $485 \mathrm{~nm}$ and emission of $520 \mathrm{~nm}$. The data represent the mean of four experiments.

Electric cell-substrate impedance sensing (ECIS) analysis. The transendothelial electrical resistance (TEER) across a monolayer of HUVECs was measured using the ECIS technique (ECIS Z $\theta$; Applied BioPhysics, Troy, NY, USA) and were analyzed using the integrated ECIS software (12). Briefly, HUVECs were plated in ECIS 8W10E+ arrays and allowed to grow to $100 \%$ confluence. Following catalpol treatment, the resistance across the EC layer was determined every $8 \mathrm{sec}$ by measuring the alternating current through the cells using electrodes. Data plots are representative of triplicate experiments, with each graph showing impedance readings from a separate well, at 40 distinct electrodes per well.
Western blotting. HUVECs treated with catalpol were lysed in radioimmunoprecipitation assay buffer (containing $20 \mathrm{mM}$ Tris, $\mathrm{pH}$ 7.5; $150 \mathrm{mM} \mathrm{NaCl}, 50 \mathrm{mM} \mathrm{NaF}, 1 \%$ NP40, $0.1 \%$ DOC, $0.1 \% \mathrm{SD}$ and $1 \mathrm{mM}$ EDTA; EMD Millipore, Billerica, MA, USA) supplemented with $1 \mu \mathrm{g} / \mathrm{ml}$ aprotonin (Roche Diagnostics GmbH, Mannheim, Germany), $10 \mu \mathrm{g} / \mathrm{ml}$ leupeptin (Roche Diagnostics $\mathrm{GmbH}$ ) and $1 \mathrm{mM}$ PMSF protease inhibitors (Beyotime Institute of Biotechnology, Shanghai, China). An equal quantity of protein for each sample was electrophoresed through a $10 \%$ SDS-PAGE gel (Beyotime Institute of Biotechnology) and then transferred to a nitrocellulose membrane (EMD Millipore). The membrane was blocked with $2.5 \%$ non-fat milk in Tris-buffered saline with Tween-20 (TBST; Beyotime Institute of Biotechnology) at $37^{\circ} \mathrm{C}$ for $1.5 \mathrm{~h}$ prior to being incubated with the following primary antibodies overnight at $4^{\circ} \mathrm{C}$. After washing with TBST three times, the membrane was incubated with secondary antibody at $37^{\circ} \mathrm{C}$ for $1 \mathrm{~h}$. The primary antibodies were used as follows: Rabbit polyclonal anti-VE-cadherin (1:500; ab33168), rabbit polyclonal anti-occludin (1:500; ab31721), rabbit polyclonal anti-ERG (1:1,000; ab28662) (Abcam, Cambridge, MA, USA), and rabbit monoclonal anti-glyceraldehyde 3-phosphate dehydrogenase (GAPDH; 1:2,500; \#2118) (14C10) mAb (Cell Signaling Technology, Inc., Danvers, MA, USA). The secondary antibody was horseradish peroxidase-linked anti-rabbit IgG (1:200; \#7074; Cell Signaling Technology, Inc.). The blots were developed with enhanced chemiluminescence reagents (EMD Millipore).

Immunofluorescence. HUVECs were allowed to grow to $100 \%$ confluence on fibronectin-coated glass chamber slides (Sigma-Aldrich) and were then treated with $1 \mathrm{mmol}$ catalpol. After $24 \mathrm{~h}$, the medium was aspirated and the monolayers were washed with phosphate-buffered saline (PBS), fixed with 4\% paraformaldehyde for $10 \mathrm{~min}$, and washed three times with PBS for $10 \mathrm{~min}$. Immunofluorescence was performed by staining with a primary antibody against human VE-cadherin 
(ab33168) or occludin (ab31721) (Abcam) at a dilution of 1:500 overnight at $4^{\circ} \mathrm{C}$ and a rhodamine-labeled secondary antibody (1:200; SA00007-2; Proteintech Group, Inc., Chicago, IL, USA) for $30 \mathrm{~min}$. The slides were photographed using an Olympus LCX100 Imaging system (Olympus Corporation, Tokyo, Japan) with an excitation wavelength of $546 \mathrm{~nm}$.

ERG knockdown with small interfering RNA (siRNA). siRNA against human ERG (NM_004440) and control non-targeting siRNA were purchased from GE Dharmacon (Lafayette, CO, USA). Transfections were performed according to the manufacturer's instructions. HUVECs were seeded into 6-well plates and cultured for $24 \mathrm{~h}$. Subsequently, $200 \mathrm{nM}$ siRNA in combination with $3 \mu$ l DharmaFECT4 Transfection Reagent (GE Dharmacon). were added to each well. After $24 \mathrm{~h}$, the cells were harvested for ECIS analysis. The knockdown of ERG was analyzed by RT-qPCR and immunoblot analysis.

Reverse transcription-quantitative polymerase chain reaction. Confluent HUVECs on 6-well plates were treated with $1 \mathrm{mmol}$ catalpol and collected at $24 \mathrm{~h}$. Total RNA was extracted with the RNeasy Mini kit (Qiagen, Hilden, Germany) according to the manufacturer's instructions. Reverse transcription was performed using the RevertAid First Strand cDNA synthesis kit (Thermo Fisher Scientific, Inc.). qPCR was conducted using SYBR Premix Ex TaqII (Takara Bio, Inc., Shita, Japan) on the ViiA 7 DX Real-Time PCR System (Thermo Fisher Scientific, Inc.). The sequences of primers for $E R G, C D H 5$ (encoding VE-cadherin) and $O L D N$ (encoding occludin) genes are shown in Table I. The reaction conditions were as follows: $94^{\circ} \mathrm{C}$ for $5 \mathrm{~min}, 30 \mathrm{cycles}$ of $94^{\circ} \mathrm{C}$ for $30 \mathrm{sec}, 57^{\circ} \mathrm{C}$ for $30 \mathrm{sec}$ and $72^{\circ} \mathrm{C}$ for $2 \mathrm{~min}$, then a final extension at $72^{\circ} \mathrm{C}$ for $5 \mathrm{~min}$. All PCR reactions were repeated in triplicate. Gene expression was assessed by comparing the relative expression level of each gene with the internal reference GAPDH using the ${ }^{\Delta \Delta} \mathrm{Ct}$ method.

Statistical analysis. Statistical significance was assessed using paired-sample t-tests. $\mathrm{P}<0.05$ was considered to indicate a statistically significant difference. Statistical analyses were performed using SPSS 18.0 software (SPSS, Inc, Chicago, IL, USA). All experiments were repeated three times unless otherwise stated.

\section{Results}

Catalpol induces vascular permeability in a dose-dependent manner. In order to examine the effects of catalpol on the permeability of endothelial cells, a FITC-Dextran Transwell assay was performed for HUVEC monolayers treated with different concentrations of catalpol. It was demonstrated that the FITC-Dextran passage remained unchanged following treatment with $0.01(1.13 \pm 0.08$ fold, $\mathrm{P}=0.33)$ and $0.1 \mathrm{mmol}$ catalpol $(1.22 \pm 0.15$ fold, $\mathrm{P}=0.18)$, but significantly increased with $1 \mathrm{mmol}$ catalpol treatment $(3.98 \pm 0.49$ fold, $\mathrm{P}<0.01)$ (Fig. 1A). In addition, catalpol-induced permeability was measured using an ECIS system, which allows for real-time measurements of TEER. In the ECIS circuit, current flows across confluent endothelial cells while the intercellular barrier functions as a resistor. As shown in Fig. 1B and C,

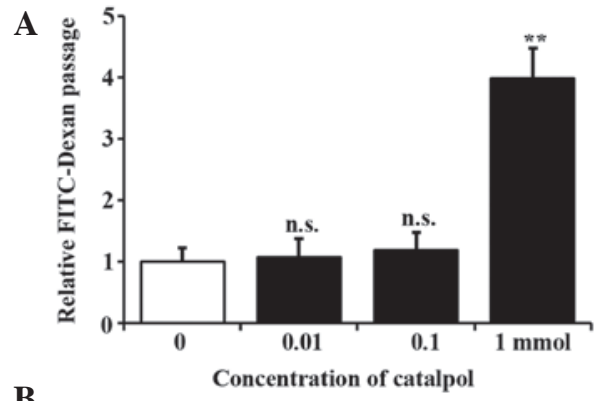

B
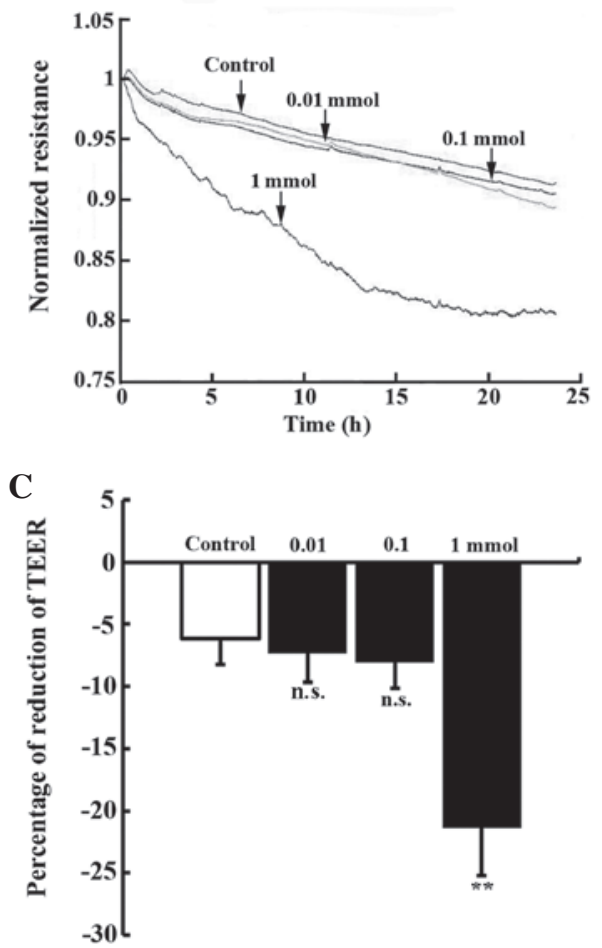

Figure 1. Catalpol induces vascular permeability. (A) FITC-Dextran Transwell assay of HUVEC monolayers treated with 0, 0.01, 0.1 and $1 \mathrm{mM}$ catalpol. (B) Real-time TEER measurement of HUVEC monolayer treated with $0,0.01,0.1$ and $1 \mathrm{mM}$. (C) Bar graph of the mean percentage of reduction of TEER. Error bars represent the standard error of the mean $(n=4)$. n.s., non-significant; ${ }^{* *} \mathrm{P}<0.01$, compared with the control. FITC, fluorescein isothiocyanate; TEER, transendothelial electrical resistance; HUVECs, human umbilical vein endothelial cells.

$1 \mathrm{mmol}$ catalpol treatment induced a significant decrease in TEER in HUVECs during the $24 \mathrm{~h}$ measurement while the TEER of the control, and $0.01 \mathrm{mmol}$ and $0.1 \mathrm{mmol}$ catalpol treatment groups remained unchanged.

Catalpol reduces the expression of VE-cadherin but not occludin. Adherens junctions and tight junctions are key in regulating vascular permeability (13). VE-cadherin and occludin are the key components of adherens junctions and tight junctions, respectively (13). The effects of catalpol on VE-cadherin and occludin expression were examined. In HUVECs treated with 0.01 or $0.1 \mathrm{mmol}$ catalpol, the levels of VE-cadherin were similar to that of non-treated cells (Fig. 2A). At a concentration of $1 \mathrm{mmol}$, catalpol significantly decreased the quantity of VE-cadherin (Fig. 2A). However, the protein levels of occludin did not show a significant change in HUVECs treated with catalpol at all concentrations (Fig. 2B). In a time course study with $1 \mathrm{mmol}$ 
A

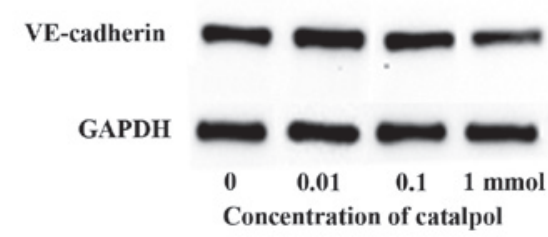

C

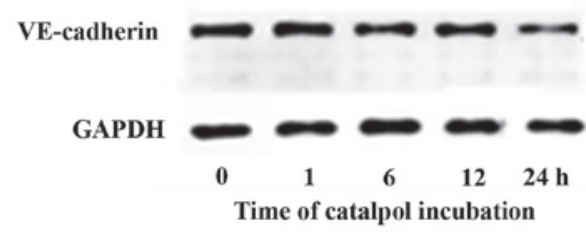

B

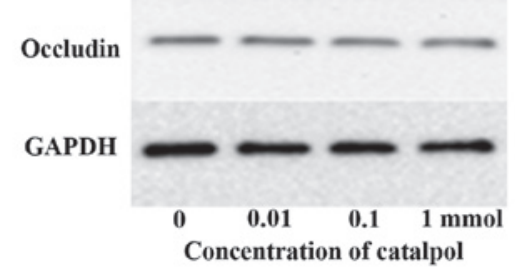

D

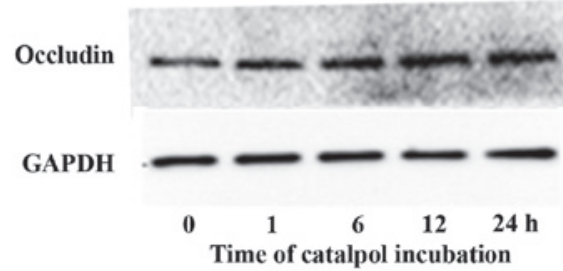

Figure 2. Effects of catalpol on the expression of VE-cadherin and occludin. Representative blots of (A) VE-cadherin and (B) occludin, and GAPDH from protein samples of HUVECs treated with catalpol at 0, 0.01, 0.1 and $1 \mathrm{mM}$ for $24 \mathrm{~h}$. Representative blots of (C) VE-cadherin and (D) occludin and GAPDH from protein samples of HUVECs treated with catalpol at a concentration of $1 \mathrm{mM}$ for $0,1,6,12$ and $24 \mathrm{~h}$. VE, vascular endothelial; GAPDH, glyceraldehyde 3-phosphate dehydrogenase; HUVECs, human umbilical vein endothelial cells.
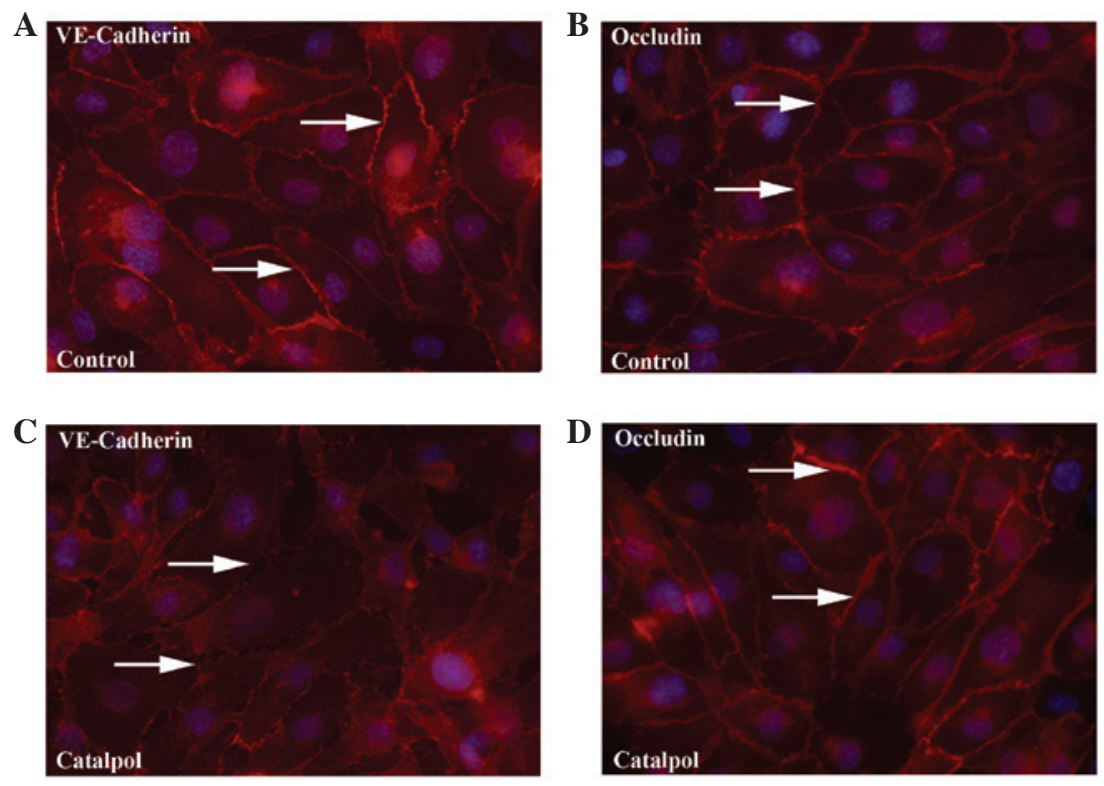

Figure 3. Localization of VE-cadherin and occludin in HUVECs treated with catalpol. Immunofluoresence staining of (A) VE-cadherin and (B) occludin on HUVEC monolayer. Immunofluoresence staining of (C) VE-cadherin and (D) occludin on the HUVEC monolayer treated with $1 \mathrm{mM}$ catalpol for $24 \mathrm{~h}$. Arrows indicate cell-cell junctions of the HUVEC monolayer; magnification, x200. VE, vascular endothelial; HUVECs, human umbilical vein endothelial cells.

catalpol, the level of VE-cadherin protein decreased over time while occludin remained unchanged (Fig. 2C and D). The expression of VE-cadherin and occludin was also examined in HUVEC monolayers by immunofluoresence. As expected, VE-cadherin and occludin were highly localized to the cell-cell contacts throughout the monolayer of non-treated HUVECs (Fig. 3A and B). Upon treatment with $1 \mathrm{mM}$ catalpol, lower levels of VE-cadherin were found to be associated with junctions while the expression of occludin was similar to that of the controls (Fig. 3C and D). VE-cadherin and occludin remained localized on the membrane of the endothelial cells, suggesting the distribution of these proteins was not affected by catalpol treatment. These results indicate that catalpol specifically reduced protein expression of VE-cadherin.
Catalpol inhibits the expression of the ERG transcription factor. The ERG (ETS-related gene) transcription factor regulates VE-cadherin expression (14). To investigate the mechanisms underlying the function of catalpol, the transcription of $E R G, C D H 5$ (encoding VE-cadherin) and $O L D N$ (encoding occludin) genes were examined in HUVECs treated with $1 \mathrm{mM}$ catalpol for $24 \mathrm{~h}$. The results of the RT-qPCR demonstrated a significant decrease in mRNA levels of ERG $(\mathrm{P}<0.01)$ and VE-cadherin $(\mathrm{P}<0.01)$, but not occludin $(\mathrm{P}=0.27)$ (Fig. 4A). The protein level of ERG was also examined in HUVECs treated with catalpol by western blot analysis. The ERG protein level was decreased by treatment with catalpol in a dose- and time-dependent manner (Fig. 4B and C). To verify the role of ERG in catalpol-induced vascular permeability, the expression of ERG was knocked down in 

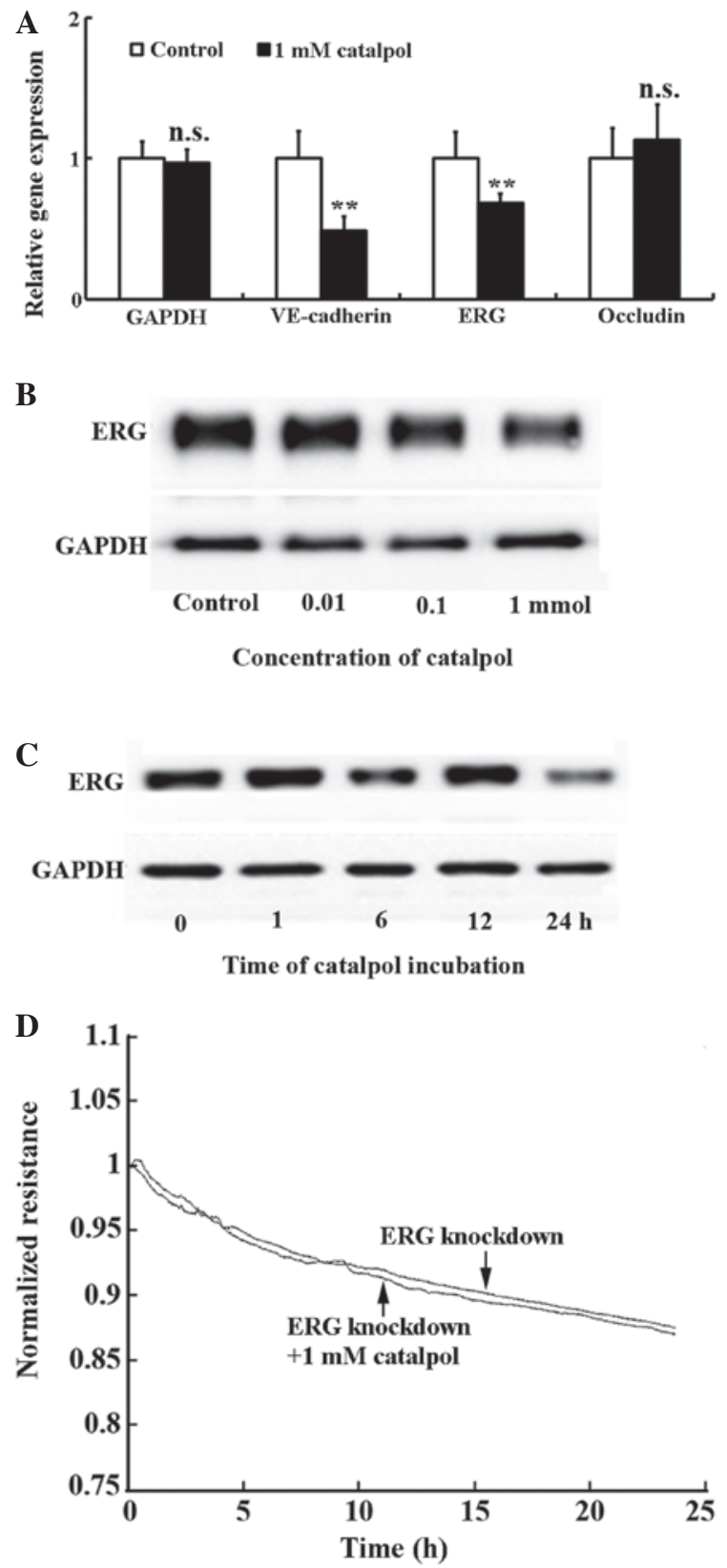

Figure 4. Catalpol inhibits the expression of the ERG transcription factor. (A) Relative VE-cadherin, ERG and occludin mRNA expression measured by reverse transcription-quantitative polymerase chain reaction in HUVECs treated with $1 \mathrm{mM}$ catalpol for $24 \mathrm{~h}$. Error bars represent the standard error of the mean $(\mathrm{n}=4)$. n.s., non-significant; ${ }^{* *} \mathrm{P}<0.01$ compared with control. Representative blots of ERG and GAPDH from protein samples of HUVECs treated with (B) catalpol at $0,0.01,0.1$ and $1 \mathrm{mM}$ for $24 \mathrm{~h}$ and $(\mathrm{C})$ at a concentration of $1 \mathrm{mM}$ for $0,1,6,12,24 \mathrm{~h}$. (D) Real-time TEER measurement of ERG-knockdown HUVEC monolayer in the absence or presence of $1 \mathrm{mM}$ catalpol. VE, vascular endothelial; HUVECs, human umbilical vein endothelial cells; GAPDH, glyceraldehyde 3-phosphate dehydrogenase; TEER, transendothelial electrical resistance.

HUVECs using siRNA, and these cells were subsequently plated as monolayers for examination of permeability using the ECIS system. In the ERG-deficient cells, no significant change in TEER was observed in the absence or presence of $1 \mathrm{mM}$ catalpol at any time point during a $24-\mathrm{h}$ incubation (Fig. 4D). Thus, this demonstrated that catalpol increases vascular permeability via downregulation of the ERG transcriptional factor.

\section{Discussion}

Catalpol has been widely used in traditional Chinese medicine (15). Similar to other drugs, catalpol enters the circulation and interacts with the vascular endothelial cells that line the blood vessels. In the present study, the effects of catalpol on endothelial cells were analyzed, and it was demonstrated that catalpol induces vascular permeability in a dose-dependent manner. In addition, catalpol significantly inhibits the expression of VE-cadherin but not occludin. The ERG transcription factor, a positive regulator of VE-caherin expression, was downregulated by catalpol. Knockdown of ERG expression compromised catalpol-induced hyperpermeability in HUVECs. From these results, it was concluded that catalpol induces the downregulation of the ERG transcription factor, which decreases VE-cadherin expression and increases vascular permeability.

Increased vascular permeability is a key pathophysiologic event associated with inflammation (1). Previous studies reported the anti-inflammatory effects of catalpol $(8,9,16)$; however, its effects on the vascular system have not yet been investigated. The present study demonstrated an increase in permeability of HUVEC monolayers in response to $1 \mathrm{mM}$ catalpol using a Transwell permeability assay and ECIS measurement. This effective dose is consistent with previous dose-response studies of catalpol, wherein concentrations of 0.5-1 mM catalpol affected cellular function (16,17). Catalpol may reduce the inflammatory response by inhibiting the expression of pro-inflammatory cytokines and proteins, such as inducible nitric oxide synthase, cyclooxygenase- 2 and Toll-like receptor 4 (16). However, the catalpol-induced hyperpermeability may exaggerate the inflammatory responses of the endothelium and increase the passage of plasma proteins. This study determined the previously unknown effects of catalpol on vascular endothelium and suggests a pro-inflammatory role.

VE-cadherin is specifically expressed in endothelial cells and is the major component of adherens junctions (18). In this study, it was demonstrated that catalpol decreases the mRNA and protein level of VE-cadherin but not occludin, suggesting that catalpol specifically disrupts adherens junctions to increase vascular permeability. The expression and distribution of VE-cadherin is tightly regulated by the microenvironment of the endothelium $(19,20)$. Extracellular stimuli, such as TNF- $\alpha$, thrombin and cadmium, increase vascular permeability by disruption of the homophilic interaction of VE-cadherin $(18,21,22)$. Other factors, such as basic fibroblast growth factor, may enhance VE-cadherin expression (23). In this study, it was identified that catalpol significantly inhibits the mRNA and protein level of VE-cadherin. In a rat model of stroke, catalpol increases infarcted-brain angiogenesis by upregulating vascular endothelial growth factor (VEGF) expression (24). VEGF, also known as a vascular permeability factor, is a potent enhancer of microvascular permeability (25). In addition, VEGF directly inhibits VE-cadherin expression, and induces its phosphorylation and internalization in endothelial cells (26-28). Thus, increased VEGF may contribute to catalpol-induced inhibition of VE-cadherin.

The ETS family member ERG is specifically and constitutively expressed in endothelial cells (29). The ERG 
transcription factor drives the expression of genes involved in endothelial homeostasis and angiogenesis (30). ERG binds to the VE-cadherin promoter and enhances its activity (14). Inhibition of ERG expression by siRNA in HUVECs also decreased the expression of VE-cadherin. In this study, it was demonstrated that catalpol inhibits the mRNA and protein expression of ERG, suggesting that catalpol is an inhibitor of ERG. During inflammation, TNF- $\alpha$ downregulates ERG expression in endothelial cells, which subsequently modifies the expression of genes mediating inflammatory responses, including IL-8, intracellular adhesion molecule-2, von Willibrand factor and VE-cadherin (14,30-32). In this context, catalpol may have a similar pro-inflammatory effect on endothelial cells. Moreover, catalpol failed to induce additional reduction of TEER in HUVECs following knockdown of ERG. This confirmed that ERG transcription factor mediates catalpol-induced vascular permeability.

In conclusion, it was demonstrated that catalpol increases vascular permeability in HUVEC monolayers. Catalpol inhibits the expression of the junctional molecule VE-cadherin, but not occludin. In addition, catalpol downregulates the expression of the ERG transcription factor, which mediates catalpol-induced hyperpermeability. These results are important for the further exploration of the clinical potential of catalpol.

\section{Acknowledgements}

This study was supported by the grants from Science and Technology Development Plan of Shandong Province (grant no. 2013GSF11805) and the Shandong Taishan Scholarship (awarded to Professor Ju Liu).

\section{References}

1. Phillipson $\mathrm{M}$ and Kubes P: The neutrophil in vascular inflammation. Nat Med 17: 1381-1390, 2011.

2. Popović M, Smiljanić K, Dobutović B, Syrovets T, Simmet T and Isenović ER: Thrombin and vascular inflammation. Mol Cell Biochem 359: 301-313, 2012.

3. Lindbom L: Regulation of vascular permeability by neutrophils in acute inflammation. Chem Immunol Allergy 83: 146-166, 2003.

4. Aghajanian A, Wittchen ES, Allingham MJ, Garrett TA and Burridge K: Endothelial cell junctions and the regulation of vascular permeability and leukocyte transmigration. J Thromb Haemost 6: 1453-1460, 2008.

5. Vestweber D: VE-cadherin: The major endothelial adhesion molecule controlling cellular junctions and blood vessel formation. Arterioscler Thromb Vasc Biol 28: 223-232, 2008.

6. Van Itallie CM and Anderson JM: Architecture of tight junctions and principles of molecular composition. Semin Cell Dev Biol 36: 157-165, 2014.

7. Wang Z, An LJ, Duan YL, Li YC and Jiang B: Catalpol protects rat pheochromocytoma cells against oxygen and glucose deprivation-induced injury. Neurol Res 30: 106-112, 2008.

8. Zhang X, Jin C, Li Y, Guan S, Han F and Zhang S: Catalpol improves cholinergic function and reduces inflammatory cytokines in the senescent mice induced by D-galactose. Food Chem Toxicol 58: 50-55, 2013.

9. Fu K, Piao T, Wang M, Zhang J, Jiang J, Wang X and Liu H: Protective effect of catalpol on lipopolysaccharide-induced acute lung injury in mice. Int Immunopharmacol 23: 400-406, 2014.

10. Liu YR, Li PW, Suo JJ, Sun Y, Zhang BA, Lu H, Zhu HC and Zhang GB: Catalpol provides protective effects against cerebral ischaemia/reperfusion injury in gerbils. J Pharm Pharmacol 66: 1265-1270, 2014.
11. Tian YY, An LJ, Jiang L, Duan YL, Chen J and Jiang B: Catalpol protects dopaminergic neurons from LPS-induced neurotoxicity in mesencephalic neuron-glia cultures. Life Sci 80: 193-199, 2006.

12. Hong J, Kandasamy K, Marimuthu M, Choi CS and Kim S: Electrical cell-substrate impedance sensing as a non-invasive tool for cancer cell study. Analyst 136: 237-245, 2011.

13. Wallez Y and Huber P: Endothelial adherens and tight junctions in vascular homeostasis, inflammation and angiogenesis. Biochim Biophys Acta 1778: 794-809, 2008.

14. Yuan L, Le Bras A, Sacharidou A, Itagaki K, Zhan Y, Kondo M, Carman CV, Davis GE, Aird WC and Oettgen P: ETS-related gene (ERG) controls endothelial cell permeability via transcriptional regulation of the claudin 5 (CLDN5) gene. J Biol Chem 287: 6582-6591, 2012.

15. Li DQ, Bao YM, Li Y, Wang CF, Liu Y and An LJ: Catalpol modulates the expressions of $\mathrm{Bcl}-2$ and $\mathrm{Bax}$ and attenuates apoptosis in gerbils after ischemic injury. Brain Res 1115: 179-185, 2006.

16. Bi J, Jiang B, Zorn A, Zhao RG, Liu P and An LJ: Catalpol inhibits LPS plus IFN-gamma-induced inflammatory response in astrocytes primary cultures. Toxicol In Vitro 27: 543-550, 2013.

17. Bi J, Jiang B, Liu JH, Lei C, Zhang XL and An LJ: Protective effects of catalpol against $\mathrm{H} 2 \mathrm{O} 2$-induced oxidative stress in astrocytes primary cultures. Neurosci Lett 442: 224-227, 2008.

18. Hordijk PL, Anthony E, Mul FP, Rientsma R, Oomen LC and Roos D: Vascular-endothelial-cadherin modulates endothelial monolayer permeability. J Cell Sci 112: 1915-1923, 1999.

19. Dejana E and Giampietro C: Vascular endothelial-cadherin and vascular stability. Curr Opin Hematol 19: 218-223, 2012.

20. Gavard J: Breaking the VE-cadherin bonds. FEBS lett 583: 1-6, 2009.

21. Yuan SY: Protein kinase signaling in the modulation of microvascular permeability. Vascul Pharmacol 39: 213-223, 2002.

22. Dong F, Guo F, Li L, Guo L, Hou Y, Hao E, Yan S, Allen TD and Liu J: Cadmium induces vascular permeability via activation of the p38 MAPK pathway. Biochem Biophys Res Commun 450: 447-452, 2014

23. Prandini MH, Dreher I, Bouillot S, Benkerri S, Moll T and Huber P: The human VE-cadherin promoter is subjected to organ-specific regulation and is activated in tumour angiogenesis. Oncogene 24: 2992-3001, 2005.

24. Zhu HF, Wan D, Luo Y, Zhou JL, Chen L and Xu XY: Catalpol increases brain angiogenesis and up-regulates VEGF and EPO in the rat after permanent middle cerebral artery occlusion. Int J Biol Sci 6: 443-453, 2010

25. Dvorak HF: VPF/VEGF and the angiogenic response. Semin Perinatol 24: 75-78, 2000.

26. Hebda JK, Leclair HM, Azzi S, Roussel C, Scott MG, Bidère N and Gavard $\mathrm{J}$ : The $\mathrm{C}$-terminus region of $\beta$-arrestin1 modulates VE-cadherin expression and endothelial cell permeability. Cell Commun Signal 11: 37, 2013.

27. Gavard J and Gutkind JS: VEGF controls endothelial-cell permeability by promoting the beta-arrestin-dependent endocytosis of VE-cadherin. Nat Cell Biol 8: 1223-1234, 2006.

28. Esser S, Lampugnani MG, Corada M, Dejana E and Risau W: Vascular endothelial growth factor induces VE-cadherin tyrosine phosphorylation in endothelial cells. J Cell Sci 111: 1853-1865, 1998.

29. Oettgen P: Regulation of vascular inflammation and remodeling by ETS factors. Circ Res 99: 1159-1166, 2006.

30. Sperone A, Dryden NH, Birdsey GM, Madden L, Johns M, Evans PC, Mason JC, Haskard DO, Boyle JJ, Paleolog EM and Randi AM: The transcription factor Erg inhibits vascular inflammation by repressing NF-kappaB activation and proinflammatory gene expression in endothelial cells. Arterioscler Thromb Vasc Biol 31: 142-150, 2011.

31. Yuan L, Nikolova-Krstevski V, Zhan Y, Kondo M, Bhasin M, Varghese L, Yano K, Carman CV, Aird WC and Oettgen P: Antiinflammatory effects of the ETS factor ERG in endothelial cells are mediated through transcriptional repression of the interleukin-8 gene. Circ Res 104: 1049-1057, 2009.

32. Liu J, Yuan L, Molema G, Regan E, Janes L, Beeler D, Spokes KC, Okada Y, Minami T, Oettgen P and Aird WC: Vascular bed-specific regulation of the von Willebrand factor promoter in the heart and skeletal muscle. Blood 117: 342-351, 2011. 